\title{
Coif-9408127--6
}

UCRL-JC-118752

PREPRINT

\section{Laser Plasma Interactions in Hohlraums}

\author{
W. L. Kruer
}

This paper was prepared for submittal to the 45th Scottish Universities Summer School in Physics

Laser-Plasma Interactions

St. Andrews, Scotland

August 7-13, 1994

October 5, 1994

This is a preprint of a paper intend ed for publication in a journal or proceedings. Since changes may be made before publication, this preprint is made available with the understanding that it will not be cited or reproduced without the permission of the author.

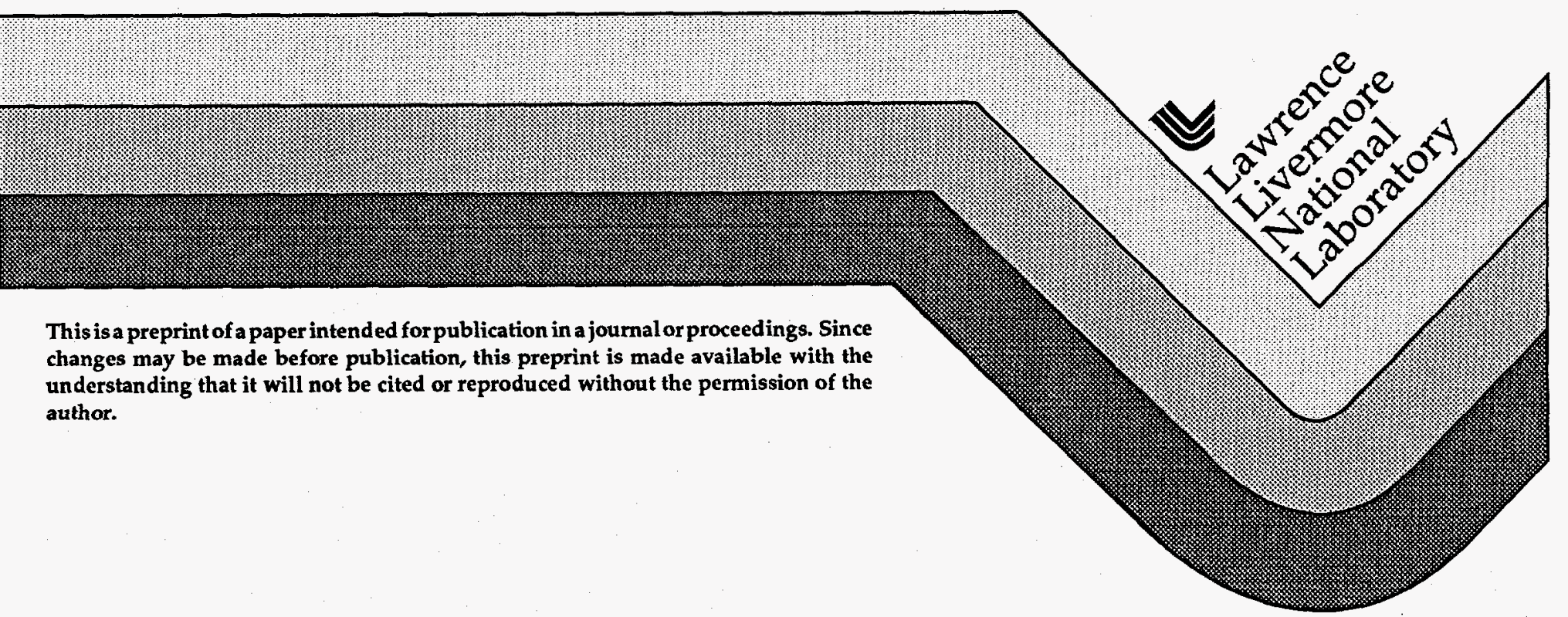<smiles>[AlH2]</smiles> 


\section{DISCLAIMER}

This document was prepared as an account of work sponsored by an agency of the United States Government. Neither the United States Government nor the University of California nor any of their employees, makes any warranty, express or implied, or assumes any legal liability or responsibility for the accuracy, completeness, or usefulness of any information, apparatus, product, or process disclosed, or represents that its use would not infringe privately owned rights. Reference herein to any specific commercial product, process, or service by trade name, trademark, manufacturer, or otherwise, does not necessarily constitute or imply its endorsement, recommendation, or favoring by the United States Government or the University of California. The views and opinions of authors expressed herein do not necessarily state or reflect those of the United States Government or the University of California, and shall not be used for advertising or product endorsement purposes. 


\section{DISCLAIMER}

Portions of this document may be illegible in electronic image products. Images are produced from the best available original document. 


\title{
Laser Plasma Interactions in Hohlraums
}

\author{
William L. Kruer \\ Lawrence Livermore National Laboratory
}

\begin{abstract}
$\underline{\text { Abstract }}$
Lasers plasma instabilities are an important constraint in $\mathrm{x}$-ray driven inertial confinement fusion. In hohlraums irradiated with $1.06 \mu \mathrm{m}$ light on the Shiva laser, plasma instabilities were extremely deleterious, driving the program to the use of shorter wavelength light. Excellent coupling has been achieved in hohlraums driven with $0.35 \mu \mathrm{m}$ light on the Nova laser.

Considerable attention is being given to the scaling of this excellent coupling to the larger hohlraums for an ignition target. Various instability control mechanisms such as large plasma wave damping and laser beam incoherence are discussed, as well as scaling experiments to check the instability levels.
\end{abstract}

\section{Introduction}

In $\mathrm{x}$-ray driven ${ }^{1-3}$ inertial fusion, laser light is converted to $\mathrm{x}$-rays in a hohlraum target. As shown in Figure 1, a hohlraum is simply a cylindrical can made of Au or some other high Z material with entrance holes for the laser beams. The laser beams efficiently heat the high $\mathrm{Z}$ walls, which efficiently radiate. These $\mathrm{x}$-rays in turn heat the portion of the hohlraum walls not directly illuminated, finally producing an environment in which a small capsule within the hohlraum is quite symmetrically driven by $\mathrm{x}$-rays. Advantages of the $\mathrm{x}$-ray drive approach include drive symmetrization and increased ablation efficiency. Disadvantages include an energy penalty due to $\mathrm{x}$-ray conversion and wall losses, as well as increased susceptibility to laser plasma instabilities.

Collisional absorption of laser light is very efficient in the dense, high $\mathrm{Z}$ plasma near the wall. It is instructive to examine the inverse bremssthralung absorption length as a function of density. We assume $0.35 \mu \mathrm{m}$ light, a characteristic electron temperature of $3 \mathrm{keV}$, and two charge 
states: $Z=50$ representative of gold plasma and $Z=2$ representative of a low $Z(\mathrm{He})$ plasma. The results are shown in Figure 2. Note that the absorption length in Au plasma is $\$ 100 \mu \mathrm{m}$ even at densities as low as $0.2 \mathrm{n}_{\mathrm{cr}}$, where $\mathrm{n}_{\mathrm{cr}}$ is the critical density. However, because of the nearly enclosed geometry, plasma blowing off the wall accumulates within the interior of the hohlraum. For typical Nova hohlraums, this plasma reaches a density of about $0.1 \mathrm{n}_{\mathrm{cr}}$ and has a scale length of about $1 \mathrm{~mm}$. Particular care must be taken to avoid instability generation in this large, low density channel plasma.

The principal instabilities 4 of concern are stimulated Brillouin and Raman scattering and filamentation. The stimulated scattering instabilities can be characterized as the resonant decay of an incident light wave into a scattered light wave plus an electrostatic wave. For stimulated Brillouin scattering (SBS), this electrostatic wave is an ion acoustic wave, whereas this wave is an electron plasma wave for stimulated Raman scattering (SRS). These instabilities can reduce absorption or change its location, which is a potential issue for drive symmetry. Furthermore, the electron plasma wave driven by SRS (or the related two-plasma decay instability near $0.25 \mathrm{n}_{\mathrm{cr}}$ ) can generate fast electrons, which are a potential preheat issue.

Filamentation is an instability in which spatial modulations in the laser beam intensity profile are amplified. Local enhancements in intensity lead to density depletions via either ponderomotive or thermal mechanisms. Refraction of the light into regions of lower density increases the intensity perturbation, leading to a breakup of the beam into intense filaments. It is prudent to avoid filamentation, since regions of high intensity could introduce other instabilities, change plasma conditions, impact x-ray conversion, or change the symmetry by spraying the light.

\section{Stimulated Raman Scattering in Shiva Hohlraums}

Avoiding these instabilities has proven to be very important ${ }^{4}$ for hohlraum design. Let's first consider Stimulated Raman Scattering (SRS), which proved to be so important in early 
hohlraum experiments with the Shiva laser. Indeed, the hot electron generation associated with underdense plasma instabilities, and in particular SRS, drove the Livermore program to the use of shorter wavelength light. This is the most studied instability and is readily identified via the spectrum of the scattered light that is down-shifted by an electron plasma frequency. Finally, this instability serves as an excellent example of a physics problem being identified and fixed.

Large underdense plasmas were encountered in early hohlraum experiments ${ }^{4-6}$ using the Shiva laser. In these experiments, the interior of a cylindrical gold can was irradiated with $1.06 \mu \mathrm{m}$ light introduced via holes as illustrated in Figure 1. The can dimensions were of order $1 \mathrm{~mm}$ and the laser pulse length of order 1 nsec. Plasma blew off the walls and accumulated within the can to density of order $(0.1-0.25) \mathrm{n}_{\mathrm{cr}}$ for $1.06 \mu \mathrm{m}$ light. Unfortunately, this plasma provided an ideal environment for the efficient excitation of underdense plasma instabilities, especially SRS.

In these experiments, the implosions were strongly degraded by electron preheat. Up to $50 \%$ of the incident laser energy was found to be converted into very energetic electrons. The characteristic temperature and the energy content of the hot electrons were inferred from the slope and the level of the high-energy $x$-rays generated when they radiate. Figure 3 shows a fluence of high-energy $x$-rays vs. $x$-ray energy measured from a Shiva hohlraum. The slope indicated a hot electron temperature of about $50 \mathrm{keV}$. The level indicates that about $50 \%$ of the incident laser energy was deposited into such electrons.

This strong hot electron generation is a signature of heating by electron plasma waves excited by laser-driven instabilities. Indeed, in the hohlraum experiment just discussed, Phillion measured about one-third of the incident laser energy in Raman-scattered light. 6 This scattered light is down-shifted by an electron plasma frequency. From the frequency or wavelength of the scattered light, one can infer the plasma density from which the scattering occurs. Figure 4 shows the measured (a) angular distribution and (b) wavelength spectrum of the Raman-scattered light. 
Most of the scattering is inferred to come from densities of about 0.1 to $0.2 \mathrm{n}_{\mathrm{cr}}$. At lower densities, the plasma wave associated with SRS becomes heavily Landau damped, and the instability is driven into a much weaker, kinetic regime. The spectrum also shows reduced scattering from plasma with the densities of about $0.2-0.25 \mathrm{n}_{\mathrm{cr}}$. This is the hohlraum version of the well known Raman "gap" in open target experiments. If one integrated over angles and wavelength, about $32 \%$ of the incident laser energy was collected as Raman-scattered light. This still remains a record level of SRS.

This hot electron problem was solved by using shorter wavelength laser light, which strongly reduces the excitation of underdense plasma instabilities. As we have seen, SRS is most efficient in plasma with a density of about $(0.1-0.25) \mathrm{n}_{\mathrm{cr}}$. Since the critical density varies as $1 / \lambda_{o}^{2}$, there is less plasma accumulated to the relevant densities with short wavelength light. What plasma is at these densities (say, near the walls) is both denser and cooler-therefore much more collisional. Indeed SRS can be collisionally suppressed with short wavelength laser light. The threshold intensity due to collisional damping in a Au plasma is about $5 \times 10^{14} \mathrm{~W} / \mathrm{cm}^{2}$ for $0.35 \mu \mathrm{m}$ light.

Strongly reduced hot electron generation with shorter wavelength light has been confirmed in hohlraum experiments. The first such experiments were carried out by Campbell and colleagues ${ }^{7}$ using the Argus laser, which was brought back into service with the option to frequency double and triple the $\mathrm{Nd}$ laser light. Figure 5 shows measurements of the high-energy $\mathrm{x}$-ray fluence vs. $\mathrm{x}$-ray energy in hohlraum experiments under nominally identical conditions but using $0.35,0.53$, or $1.06 \mu \mathrm{m}$ laser light. Note the impressive reduction in the hard $\mathrm{x}$-rays when shorter wavelength laser light is used. 


\section{Laser Plasma Coupling in Nova Hohlraums}

Very efficient laser plasma coupling has been demonstrated in hohlraums irradiated by $0.35 \mu \mathrm{m}$ light on the Nova laser. In these experiments, 8,9 the measured absorption efficiency is typically $>90-95 \%$. The fraction of energy into high energy electrons is $<1 \%$, as inferred from the high-energy $\mathrm{x}$-rays. The back reflectivity from SRS is $<1 \%$, that from SBS is $<10-15 \%$.

As an example, consider SRS, an instability which was very potent in hohlraums driven by $1.06 \mu \mathrm{m}$ light using the Shiva laser. In Nova hohlraums irradiated with $0.35 \mu \mathrm{m}$ light, the channel plasma density is sufficiently low that the electron plasma wave associated with the backscatter is strongly Landau damped. This heavy damping converts the instability to a weaker, kinetic version called stimulated Compton scattering (SCS) with growth rate

$$
\gamma=\frac{\mathrm{k}^{2} \mathrm{v}_{o s}^{2} \operatorname{Im} \varepsilon}{8 \omega_{s}|\varepsilon|^{2}}
$$

Here $\mathrm{k}$ is the wave number of the plasma wave, $\omega_{s}$ the frequency of the scattered light, $\mathrm{v}_{\mathrm{os}}$ the electron oscillatory velocity, and $\varepsilon$ the dielectric function of the plasma. For typical channel plasma conditions in a Nova hohlraum, the estimated gain coefficient is $<7$, which would lead to only a small level of backscatter. Indeed, Nova experiments show that the back reflectivity from this process is less than $1 \%$.

The suppression by strong Landau damping does not apply to stimulated Raman forward scatter, since the plasma wave then has a very long wavelength. However, calculations ${ }^{10}$ show that this forward scatter is very sensitive to density inhomogeneity and can be saturated at low levels from nonlinearly induced density perturbations. The nonlinear levels were tested in recent Nova experiments. ${ }^{11} \mathrm{~A}$ large region of low-density plasma was formed by irradiating a thin foil with eight Nova beams. This plasma was then irradiated with an intense interaction beam with a wavelength of $0.53 \mu \mathrm{m}$, and the forward scattering measured as a function of intensity. The 
measured levels are shown in Figure 6. At low intensities, these signals exponentiate in agreement with convective theory but then saturate at a very modest level which corresponds to a scattering efficiency of less than $1 \%$. We note that the estimated gain coefficients in these experiments were even greater than those projected in the NIF target.

To be conservative in our projections, we are also continuing to monitor other instabilities such as stimulated Brillouin scattering. In Nova hohlraum experiments 8,9 with relevant plasma conditions, less than $10 \%$ of the light is Brillouin backscattered. These experiments ${ }^{9}$ further indicate that this small scattering is associated with the higher density plasma near the walls. Indeed, when only the low-density channel plasma is irradiated, the back reflection drops to $<1 \%$. Use of random phase plates further reduces the reflection in this case but does not significantly reduce the reflection when the wall plasma is present. Experiments and analysis are ongoing to test the location and scaling of this Brillouin scattering.

\section{Scaling to Ignition Targets}

A key issue is the scaling of this favorable coupling to larger plasmas in the hohlraum for an ignition target. Figure 7 shows calculated contours of plasma density at time of peak power for (a) a standard hohlraum driven by a 2.2 ns-shaped Nova pulse and (b) for a future hohlraum driven by the shaped NIF pulse. In these hohlraums, the walls were coated with a layer of $\mathrm{CH}$. This lower $\mathrm{Z}$ plasma fills the channel, reducing the motion of the dense Au where the radiation is generated and improving time-dependent symmetry. The channel plasma densities are about the same ( $\$ 0.1$ $\mathrm{n}_{\mathrm{cr}}$ ). In both cases the peak electron temperature is about $4 \mathrm{keV}$. The principal difference is that the density scalelength $(\mathrm{L})$ is about 4 times larger in the future hohlraum $(\mathrm{L} \approx 1 \mathrm{~mm}$ for Nova and $\mathrm{L} \approx$ $4 \mathrm{~mm}$ for NIF).

Stimulated Brillouin scattering and filamentation are both potential risks in these large channel plasmas. Consider some ideal estimates based on coherent laser beams in a smoothly- 
varying plasma. The Rosenbluth gain for backscatter is $\exp \left(G_{B}\right)$, where $G_{B}=2 \pi \gamma_{0}^{2} / K^{\prime} v_{1} v_{2}$. Here $\gamma_{0}$ is the growth rate in a homogeneous plasma, $K^{\prime}$ the spatial derivative of the wave number mismatch, and $v_{1}$ and $v_{2}$ the group velocities of the unstable waves. Similarly, ponderomotive filamentation grows by $\exp G_{F}$, where $G_{F}=\frac{\pi}{4} \frac{n}{n_{c r}}\left(\frac{v_{o s}}{v_{e}}\right)^{2} \frac{L}{\lambda_{o}}$. Here $n$ is the plasma density, $v_{e}$ the electron thermal velocity, $v_{o s}$ the electron oscillatory velocity, $L$ the plasma length, and $\lambda_{0}$ the laser wavelength. For typical channel conditions $\left(\mathrm{I} \sim 2 \times 10^{15} \frac{\mathrm{W}}{\mathrm{cm}^{2}}, \lambda_{\mathrm{o}}=0.35 \mu \mathrm{m}, \frac{\mathrm{n}}{\mathrm{n}_{\mathrm{cr}}} \cong 0.1, \theta_{\mathrm{e}}\right.$ $\cong 4 \mathrm{keV}$, and $\mathrm{L}=3 \mathrm{~mm}), \mathrm{G} \sim 15$ and $\mathrm{G}_{\mathrm{B}}>20$. Such ideal estimates should only be viewed as grounds for caution. Our conservative approach is then to reduce instability growth using strong ion wave damping and/or laser beam incoherence, as well as to test the instability levels in plasma scaling experiments.

In hohlraums for an ignition target, $\theta_{\mathrm{i}} \cong \theta_{\mathrm{e}} / 2$, since the ion-electron equilibration time is about equal to the pulse length. It is appropriate to then describe the SBS using the damped ion wave model. The dispersion relation is

$$
\left[\left(\omega^{2}+i \omega v_{i}-k^{2} v_{s}^{2}\right)\left[\left(\omega-\omega_{o}\right)^{2}-(\underline{k}-\underline{k})^{2} c^{2}-\omega_{p e}^{2}\right]=k^{2} \frac{v_{o s}^{2}}{4} \omega_{p i}^{2} .\right.
$$

Here $\omega, k$, and $v_{i}$ are the frequency, wave number and energy damping rate of the ion wave, $\omega_{0}$ and $k_{0}$ are the frequency and wave number of the laser light, $v_{s}$ is the sound velocity, and $\omega_{p i}$ is the ion plasma frequency. Considering backscatter and setting $\mathrm{k}=\frac{\omega}{\mathrm{v}_{\mathrm{s}}}+\mathrm{K}$ gives $K=\gamma_{0}^{2} / c v_{1}$. The reflectivity $r$ is then $r=\exp \left(Q_{B}\right)$, where $Q_{B}=2 \gamma_{0}{ }^{2} L / c v_{i}$. Here $L$ is the plasma length and $I_{n}$ is the noise level normalized by the incident laser intensity. Note that the gain scales inversely with $v_{i}$. It is straight-forward to return to the coupled mode equations and include pump depletion ${ }^{12}$, giving $r(1-r)=I_{n}\left(\exp \left[Q_{B}(1-r)\right]-r\right\}$. For $I n=10^{-8}$ and $Q_{B}=20, r \cong 0.2$.

The Landau damping of an ion wave can be significantly increased 13,14 by the addition of protons. Consider a $\mathrm{CH}$ plasma with equal temperatures for the protons and carbon ions. The damping, due to ions near the phase velocity of the wave, is clearly enhanced by the lighter 
(therefore, faster) protons. Nonlinear effects can also be enhanced ${ }^{15,16}$ by the presence of protons. For example, consider the amplitude at which an ion wave traps particles. This amplitude is determined by the usual condition that the trapping velocity equal the phase velocity of the wave. Assuming that the phase velocity is determined mainly by the heavier (say, $\mathrm{C}$ ) ions and the protons are trapped, we estimate $\frac{2 Z_{H} e \phi}{M_{H}}=\frac{Z_{c} T e}{M_{c}}$. Here $\mathrm{Z}_{\mathrm{H}}$ and $\mathrm{M}_{\mathrm{H}}$ are the charge and mass of a proton; $\mathrm{Z}_{\mathrm{C}}$ and $\mathrm{M}_{\mathrm{C}}$ are the charge and mass of a carbon ion. The trapping amplitude $\left(\frac{e \phi}{T_{e}}\right)$ is reduced by $\frac{M_{H}}{M_{c}} \frac{Z_{c}}{Z_{H}}$ (i.e., by 2). This reduction is even greater when thermal effects are included.

Recent simulations ${ }^{16}$ show a reduction of SBS in multispecies plasmas due to both linear and nonlinear effects. In these 1-D simulations a hybrid code (particle ions, fluid electrons) is used to calculate the Brillouin back reflection as a function of the hydrogen content of an initially Carbon plasma. In these simulations, $\mathrm{I}=3 \times 10^{15} \frac{\mathrm{W}}{\mathrm{cm}^{2}}, \lambda_{\mathrm{o}}=0.35 \mu \mathrm{m}, \mathrm{n}=0.25 \mathrm{n}_{\mathrm{cr}}, \theta_{\mathrm{e}}=3 \mathrm{keV}$, $\mathrm{T}_{\mathrm{i}}=0.2 \mathrm{~T}_{\mathrm{e}}$ and $\mathrm{L}=160 \lambda_{\mathrm{o}}$. As shown in Figure 8, the reflectivity drops significantly as hydrogen is added. It should be noted that the level of the reflectivity is no doubt overestimated by 1-D simulations, since 2-D effects reduce the coherence of the interaction.

Reduction of Brillouin scattering by protons has been demonstrated in UCLA experiments ${ }^{17}$ by Clayton et al. A low density $\mathrm{N}_{2}$ plasma was irradiated with $10.6 \mu \mathrm{m}$ laser light. As shown in Figure 9, the Brillouin back reflectivity dropped sharply as hydrogen was added to the plasma. More recent experiments with NIF relevant plasmas show a similar trend, as will be mentioned later. Enhanced damping by protons will be exploited by using a mixture of helium and hydrogen in the channel of an ignition hohlraum. For $T_{i}=0.5 \mathrm{Te}, v_{2} \equiv 0.2 \omega$ for a He plasma. For an equal mixture of helium and hydrogen ${ }^{14}, v_{i} \cong 0.6 \omega$. Clearly plasma composition can be a very useful control mechanism.

The addition of temporal and spatial incoherence to the laser beams is another promising technique for instability control. ${ }^{18-20}$ Temporal incoherence corresponds to broadening the 
frequency spectrum either continuously or by dividing it into a series of lines. Spatial incoherence corresponds to broadening the wave-number spectrum. Both effects can reduce instabilities.

Even the simplest form of beam smoothing [the use of random phase plates (RPP)] reduces instability generation. The phase plates ${ }^{21}$ break the beam into speckles with a characteristic length of $\mathrm{l}_{\mathrm{sp}} \approx 8 \mathrm{f} \lambda_{0}$ and radius of $\mathrm{r}_{\perp} \approx \mathrm{f} \lambda_{0}$. Here $\mathrm{f}$ is the $\mathrm{f}$-number of the focusing lens and $\lambda_{0}$ the laser wavelength. Calculations ${ }^{22}$ show that filamentation can be suppressed if its growth length is less than $1_{s p}$. We note that filamentation is mainly driven by the ponderomotive mechanism in the lowdensity, high temperature channel. Stabilization then requires $f^{2}<\frac{1}{64} \frac{n_{c r}}{n} \frac{\theta k e V}{I_{16}{ }^{2}}$ where $I_{16}$ is the intensity in units of $10^{6} \mathrm{~W} / \mathrm{cm}^{2 \mathrm{j}}, \mathrm{n}$ the plasma density, $\theta_{\mathrm{keV}}$ the electron temperature in $\mathrm{keV}$, and $\lambda_{\mu}$ the laser wavelength in $\mu \mathrm{m}$. Side scattering may also be reduced by the small-scale transverse structure; i.e., the speckles are long but narrow. Even in the limit that the growth from all the speckles is additive, the gain for sidescatter is not large. More sophisticated forms of beam smoothing are expected to enhance instability suppression. With smoothing by spectral dispersion . (SSD), the speckle pattern changes with frequency $\Delta \omega$, which is the effective bandwidth. More potent reduction of filamentation is then expected when $\Delta \omega$ is $\geq$ the growth rate. For typical conditions, $\Delta \omega=3 \times 10^{-4} \omega_{0}$ appears sufficient.

The F3-D code is being used to quantify 22,23 the beneficial effects of beam smoothing on filamentation. This code includes both ponderomotive ${ }^{24}$ and thermal ${ }^{25}$ mechanisms. Nonlocal electron transport and a kinetic treatment of ion and electron damping are included. Random phase plates or smoothing by spectral dispersion are modeled by using appropriate boundary conditions. Typical results ${ }^{23}$ using RPP and the channel plasma conditions in Nova hohlraums are shown in Figure 10. The fraction of the beam energy $f(E)$ with intensity greater than 5 times the average intensity is plotted vs. the f-number. This fraction is a sensitive measure of the filamentation of hot spots in the beam. The dotted line shows initial values. The solid line shows results for a $0.35 \mu \mathrm{m}$ RPP beam with $\mathrm{I}=10^{15} \mathrm{~W} / \mathrm{cm}^{2}, \theta_{\mathrm{c}}=3 \mathrm{keV}$ and $\mathrm{n}-0.1 \mathrm{n}_{\mathrm{cr}}$. The large dot is an example using SSD 
with $\Delta \omega=10^{-4} \omega_{0}, \mathrm{I}=2 \times 10^{15} \mathrm{~W} / \mathrm{cm}^{2}$, and $\theta_{e}=4 \mathrm{keV}$. Note that filamentation is essentially stable for $\mathrm{f}$-numbers $<8$ for these conditions. Note also that the use of SSD gives additional suppression.

\section{Plasma Scaling Experiments}

Scaling experiments are being carried out to investigate both the stimulated scattering levels in large plasmas and the benefits of laser beam smoothing. In the initial experiments ${ }^{26} \mathrm{a}$ large region of plasma is created by irradiating a thin foil with a $0.35 \mu \mathrm{m}$ laser beam. After the expanding plasma becomes underdense, it is then irradiated with a $0.53 \mu \mathrm{m}$ interaction beam, and the Brillouin back-reflection is monitored. Plasma scalelengths are roughly $0.5 \mathrm{~mm}$ when the peak plasma density is about $0.1 \mathrm{n}_{\mathrm{cr} \text {. }}$ The peak density and laser intensity are varied to access estimated gain coefficients comparable to those expected in the future larger but more weakly driven plasmas.

Figure 11 shows typical results where the measured SBS back reflection is plotted vs. time (and the corresponding peak plasma density as inferred from the SRS spectrum). When the peak density is large $\left(z 0.2 \mathrm{n}_{\mathrm{Cr}}\right)$, the reflectivity is $5-10 \%$, even if one uses RPP or SSD. However for lower peak densities of order $0.1 \mathrm{n}_{\mathrm{Cr}}$, the reflectivity is $<1 \%$ with the RPP beam and negligible with an SSD beam.

The low levels and reductions by beam smoothing in low-density plasmas are very encouraging, since the large channel plasmas with the ignition hohlraum will be at low density $\left(\approx 0.1 \mathrm{n}_{\mathrm{cr}}\right)$. These results are consistent with other data on the beneficial effects of laser beam incoherence at many other laboratories $27-31$. For example, Figure 12 shows measurements of the stimulated Brillouin backscatter vs. laser intensity in experiments 27 at Imperial College, UK. A preformed plasma with density $\approx 0.08 \mathrm{n}_{\mathrm{cr}}$ and scalelength $\approx 500 \mu \mathrm{m}$ was irradiated with a $0.53 \mu \mathrm{m}$ laser beam. Note that the peak reflectivity is $\$ 1 \%$, even with an ordinary laser beam. When laser 
beam incoherence was added via the technique of induced spatial incoherence (ISI), the reflectivity dropped to the threshold of detection.

Finally, additional experiments are ongoing using larger plasmas and higher f-number lenses. These experiments are accessing plasmas with scalelengths $\sim 1-2 \mathrm{~mm}$. Various techniques are being used, including gas bags ${ }^{32}$ and specially-designed hohlraums. Results, to date $8,9,33-35$ are extremely encouraging, showing low levels of backscattering in plasmas with hydrogen and strong instability suppression by use of SSD. As an example, consider experiments 33 in which a gas bag is irradiated by nine heater beams to form a plasma with density of about $10^{21} \mathrm{~cm}^{-3}$ and electron temperature of about $3 \mathrm{keV}$. The tenth beam of Nova is then used as an interaction beam to test the SBS as a function of intensity. Plasma composition and the f-number of the focusing lens are also varied. Random phase plates are used on the interaction beam. In some experiments temporal smoothing was included using either SSD or 4-colors. In the 4-colors scheme, four beams each with a slightly different frequency are overlapped.

Measurements of the time-integrated SBS into an $\mathrm{f} / 4$ lens versus intensity are shown in Figure 13. With $\mathrm{CO}_{2}$ or $\mathrm{C}_{5} \mathrm{D}_{12}$ gas, the reflectivity can be sizable ( $\left.10-20 \%\right)$. For plasma with hydrogen (i.e. $\mathrm{C}_{5} \mathrm{H}_{12}$ ), the reflectivity is reduced by an order of magnitude. It should be noted that the peak time-dependent reflectivity is roughly several times larger. In experiments with the f/8 lens and the $\mathrm{C}_{5} \mathrm{H}_{12}$ plasma, a peak reflectivity of only about $1-2 \%$ is measured. However, preliminary measurements indicate that several times more light is scattered outside the lens. The use of 4-colors does not significantly reduce the reflectivities, although the use of SSD does. Similar results are obtained in gas-filled hohlraums. ${ }^{9,}$, 34-35

The magnitude of the reflectivity and its scaling with intensity underscore the importance of nonlinear effects, as does also the theory of the SBS of a random phase plate beam. ${ }^{21}$ 3-D linear calculations ${ }^{23}$ predict much higher reflectivities than observed unless a nonlinear damping is 
included to limit the amplitude $\delta$ of the ion fluctuations to a level $\frac{\delta n}{n} \leq 5 \%$. Both particle simulations and nonlinear theory show that kinetic effects ${ }^{15-16}$ such as trapping and modification of the ion distribution functions are important effects. The 1-D kinetic model using particle ions and fluid electrons is now being extended to two dimensions in order to include processes such as nonlinear spreading in angle of the ion waves. There is also ongoing work to better understand the beneficial effects of SSD as well as the role of filamentation, which could spray ${ }^{36}$ the laser beam into a larger spot.

\section{Summary}

In summary, advances in the understanding of laser plasma interactions continue to be a high leverage issue in laser fusion. The laser intensity for a high gain target is bound from above by laser plasma instabilities and from below by Rayleigh-Taylor instability in the capsule implosion. Laser plasma instabilities which were a serious problem in early experiments with the Shiva laser have been successfully controlled by using shorter wavelength light. Other important control mechanisms including large plasma wave damping and the use of spatial and temporal incoherence have been tested. Extrapolation of the coupling to future targets with larger plasmas looks quite favorable. More experiments and calculations are ongoing to delineate in greater detail the regimes of acceptable coupling. This improved understanding can allow more confident extrapolation to high-gain targets and may even reveal new possibilities, such as the generation of higher hohlraum temperatures or the use of $0.53 \mu \mathrm{m}$ light for directly-driven targets.

Finally, the new regime of ultra intense laser plasma interactions may also allow new possibilities for inertial fusion. In the so-called fast ignitor ${ }^{37}$ approach, $\mathrm{MeV}$ electrons generated by ultra intense pulses of laser light are used to ignite a portion of precompressed fuel. As currently envisioned, this scheme uses an intense hole-boring laser beam to prepare a channel for the ignitor pulse. In principal, there could be many advantages, such as much higher target gains and 
implosions which are less demanding with respect to implosion symmetry and stability. This new regime is under active study in order to evaluate fast ignitor options as well as other novel applications.

Acknowledgments: I am grateful for valuable discussions with numerous colleagues, including S. Wilks, M. Campbell, S. Batha, R. Berger, R.P. Drake, J. Fernandez, D. Hinkel, W. Hsing, B. Kauffman, B. Langdon, B. Lasinski, B. MacGowan, D. Montgomery, L. Powers, M. Tabak, R. Turner, E. Williams. Work performed under the auspices of the U.S. Department of Energy by the Lawrence Livermore National Laboratory under Contract W-7405-Eng-48. 


\section{References}

1. Nuckolls J H, 1982, Phys Today 35 24; Lindl J D, 1991, From Fusion to Light Surfing 177-190 ed Katsouleas T (Addison-Wesley, Redwood City, CA).

2. Kilkenny J D et al., 1989, Plasma Physics and Controlled Fusion Research 329 (IAEA, Vienna); Hauer A, 1994, Rev Sci Instruments (in press).

3. Sigel R et al., 1990, Phys Rev Lett 65 587; Nakai S et al., 1991, Laser Interaction and Related Plasma Phenomena 9 eds Hora H and Miley G H (Plenum, New York).

4. $\quad$ Kruer W L, 1991, Phys. Fluids B3 2356.

5. Ze F et al., 1986, Comments Plasma Phys, Controlled Fusion 1033.

6. Phillion $\mathrm{D}$, to be published.

7. Campbell E M, 1992, Phys Fluids B4 3781.

8. Kauffman R L et al., 1994, Lawrence Livermore National Laboratory UCRL-JC-117046.

9. Fernandez J C et al., 1994, 24th Anomalous Absorption Conference and earlier work BO-8.

10. Kruer W L, 1993, Laser Interaction and Related Plasma Phenomena 10503 (Plenum, New York).

11. Batha S H et al., 1991, Phys Rev Lett 662324.

12. Tang G L, 1966, J Appl Phys 372945.

13. Fried B D, 1971, Phys Fluids 142338.

14. Williams E A et al., 1994, 24th Anomalous Absorption Conference DP-12.

15. Kruer W L, Wilks S C, Hinkel D, and Williams E A, 1994, 24th Anomalous Absorption Conference AP-12.

16. Wilks S C et al., 1994, Lawrence Livermore National Laboratory UCRL-JC-117313.

17. Clayton C et al., 1981, Phys Fluids 242312.

18. Lehmberg R H and Obenschain S O, 1993, Opt Commun 4627.

19. Skupsky S, Short R W, Kessler T, Craxton R S, Letzring S, and Soures J M, 1989, J Appl Phys 663456.

20. Kato Y, Mima K, Miyanaga N, Arinaga S, Kitagawa Y, Nakatsuka M, and Yamanaka C, 1984, Phys Rev Lett 53 1057; Deng X, Liang X, Chen Z, Yu W, Ma R, and Chin J, 1985, Lasers 12257. 
21. Rose H A and DuBois D, 1993, Phys Fluids B5 59; Rose H A and DuBois D, 1994, Phys Rev Lett 722883.

22. Berger, R, Lasinski B F, Kaiser T B, Williams E A, Langdon A B, and Cohen B I, 1993, Phys Fluids B5.

23. Lasinski B F et al., 1994, 24th Anomalous Absorption Conference AP-14.

24. Schmitt A, 1988, Phys Fluids 313079.

25. Epperlein E, 1990, Phys Rev Lett 652145.

26. Baldis H, Montgomery D, Moody J, Labaune C, Batha S, Estabrook K, Berger R, and Kruer W, 1992, Plasma Phys Cont Fusion 342077.

27. Cole S et al., 1989, Europhys Lett. 1031.

28. Obenschain S, Pawley C, Mostovych A, Stamper J, Gardner J, Schmitt A and Bodner S, 1989, Phys Rev Lett 62 768-771.

29. Mostovych A et al., 1987, Phys Rev Lett 591193.

30. Seka W, Bahr R, Short R, Simon A, Craxton R, Montgomery D, and Rubenchik A, 1992, Phys Fluids B4 2232-2240.

31. Kato Y et al., 1984, Phys Rev Lett 531057.

32. Denavit J and Phillion D, 1994, Phys Plasma 11971.

33. MacGowan B et al., 1994, 24th Anomalous Absorption Conference AO-1.

34. Hsing W et al., 1994, 24th Anomalous Absorption Conference BO-7.

35. Turner R et al., 1994, 24th Anomalous Absorption Conference BO-6.

36. Wilks S, Young P, Hammer J, Tabak M, and Kruer W, 1994, Lawrence Livermore National Laboratory, UCRL-JC, Phys Rev. Lett (in press).

37. Tabak M et al., 1994, Phys Plasmas 1 1620; Wilks S C, Kruer W L, Tabak M, and Langdon A B, 1992, Phys Rev Letters 691383. 


\section{Figure Captions}

Fig. 1. A schematic of a hohlraum irradiated with laser beams.

Fig. 2. A representative plot of the collisional absorption length vs. plasma density for two different ion charge states. A laser wavelength of $0.35 \mu \mathrm{m}$ and an electron temperature of $3 \mathrm{keV}$ are assumed. Strong absorption limits the interaction length in Au plasma.

Fig. 3. The measured fluence of the high-energy $x$-rays vs. the $x$-ray energy in a representative Shiva hohlraum experiment.

Fig. 4. Measurements of (a) the angular distribution and (b) the wavelength spectrum of the light that is Raman scattered in the hohlraum experiment referred to in the previous figure (shot number 81082404).

Fig. 5. The measured fluence of high-energy $x$-rays vs. $x$-ray energy for irradiation with 0.35 , 0.53 , and $1.06 \mu \mathrm{m}$ laser light. These hohlraum targets were irradiated with the Argus laser.

Fig. 6. Gain factor calculated from the measured fluence levels vs. incident laser beam intensity. The peak plasma density is about $0.02 \mathrm{n}_{\mathrm{cr}}$.

Fig. 7. Contours of plasma density at time of peak power for (a) a scale 1 hohlraum irradiated with a 2.2 ns-shaped Nova pulse and (b) a hohlraum for the ignition target irradiated with a shaped NIF pulse.

Fig. 8. The SBS reflectivity as a function of the percentage of hydrogen present in the plasma.

These results are from a hybrid code with $\frac{\mathrm{n}}{\mathrm{n}_{\mathrm{cr}}}=0.25, \theta=3 \mathrm{kev}, \theta_{\mathrm{i}} / \theta_{\mathrm{i}}=0.2, \mathrm{I}=2.8 \times 10^{15} \frac{\mathrm{W}}{\mathrm{cm}^{2}}$, $\lambda_{0}=0.35 \mu \mathrm{m}$ and $\mathrm{L}=160 \lambda_{0}$.

Fig. 9. Brillouin power reflectivity at full input power vs. concentration of hydrogen added to pure nitrogen. Relfectivities are normalized to the reflectivity in pure nitrogen..

Fig. 10. The fraction of the energy with intensity $>5$ times the average intensity vs. the f-number of the lens as calculated with the F3D code.

Fig. 11. The Brillouin back reflection of a $0.53 \mu \mathrm{m}$ interaction beam vs. time from an exploding foil target. The lower scale denotes the peak plasma density inferred from the SRS spectrum. 
Fig. 12. The Brillouin back reflection into a $\mathrm{f} / 10$ lens vs. intensity for a $0.53 \mu \mathrm{m}$ beam irradiating a preformed plasma. Beam incoherence has been shown to reduce instability generation at many laboratories.

Fig. 13. The time-averaged SBS reflectivity into an f/4 lens from experiments with gas bags irradiated with $0.35 \mu \mathrm{m}$ light. 


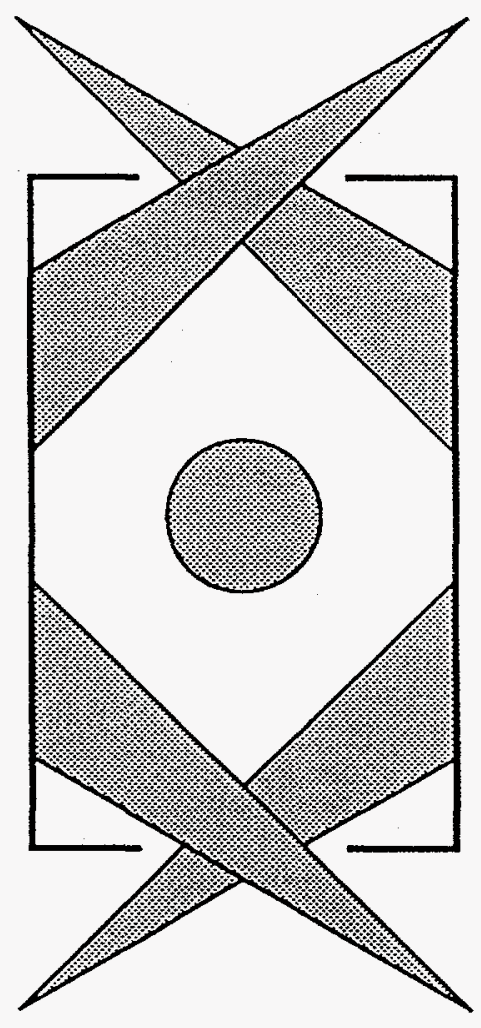




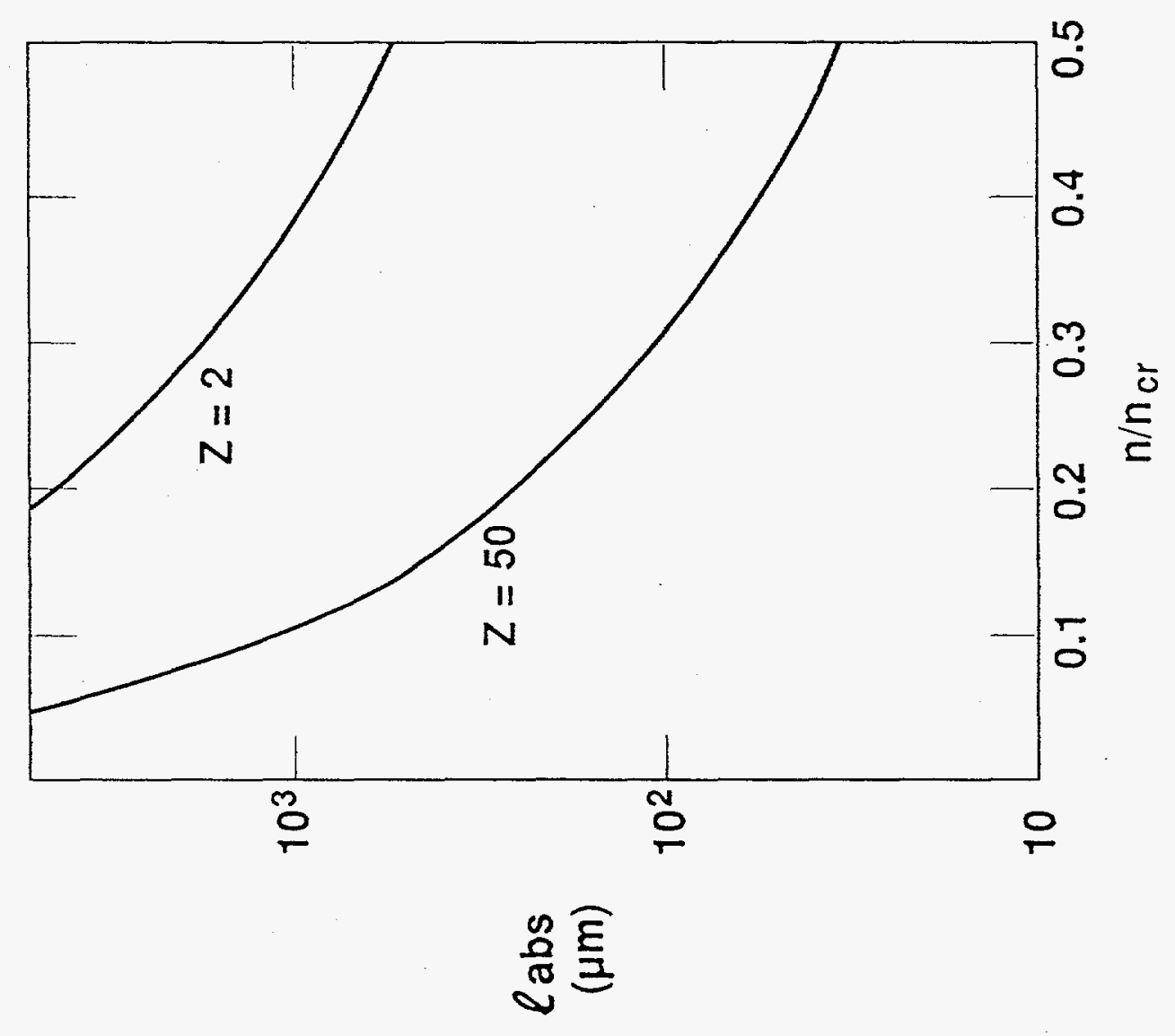

$\frac{1}{\dot{3}}$
$\frac{1}{0}$
$\frac{5}{5}$
$\frac{7}{0}$ 


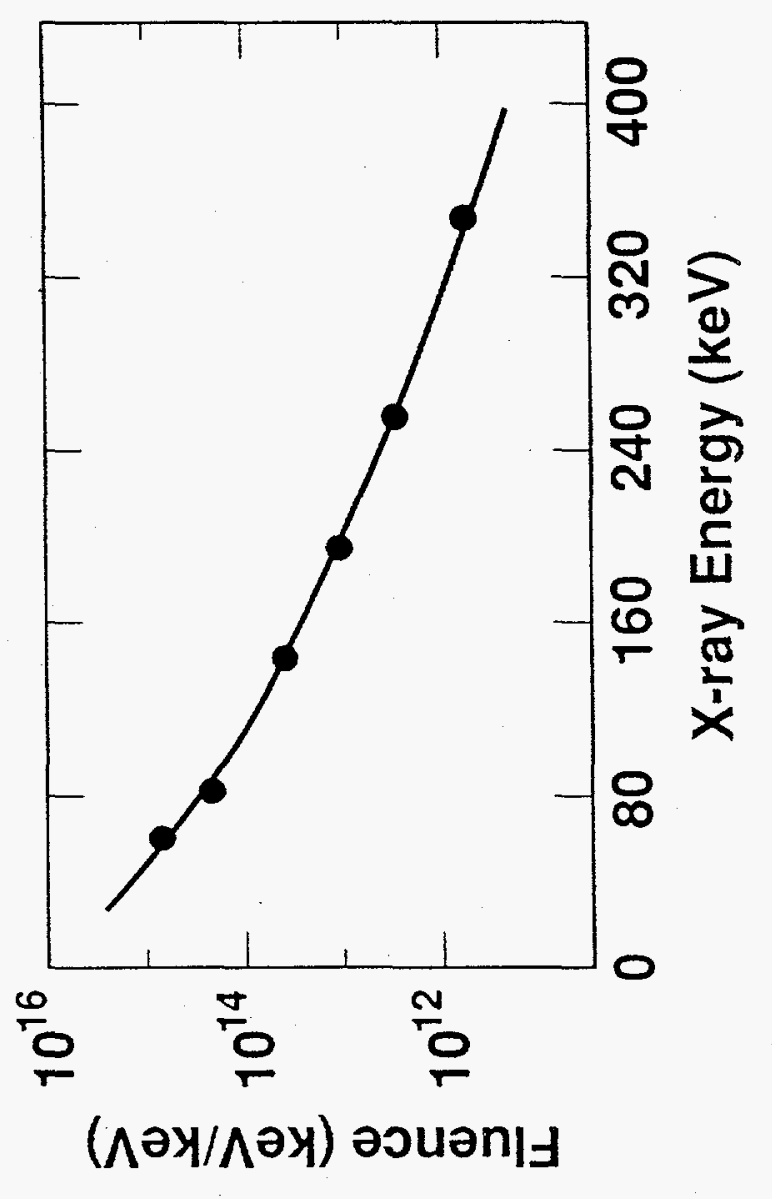

$\frac{5}{\dot{y}}$
$\frac{5}{0}$
$\frac{5}{5}$
$\frac{5}{0}$ 

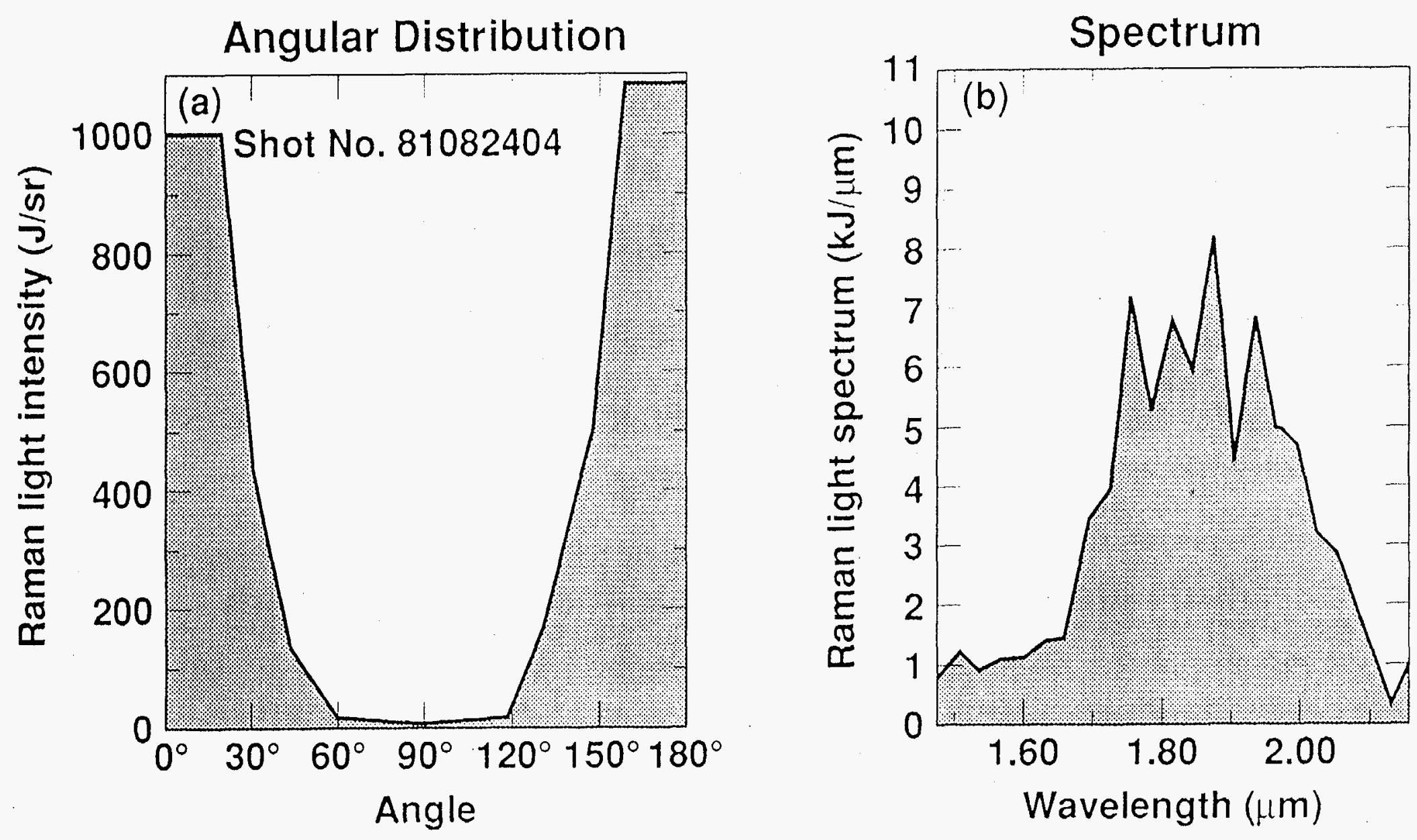

01/14/91 BK-12 


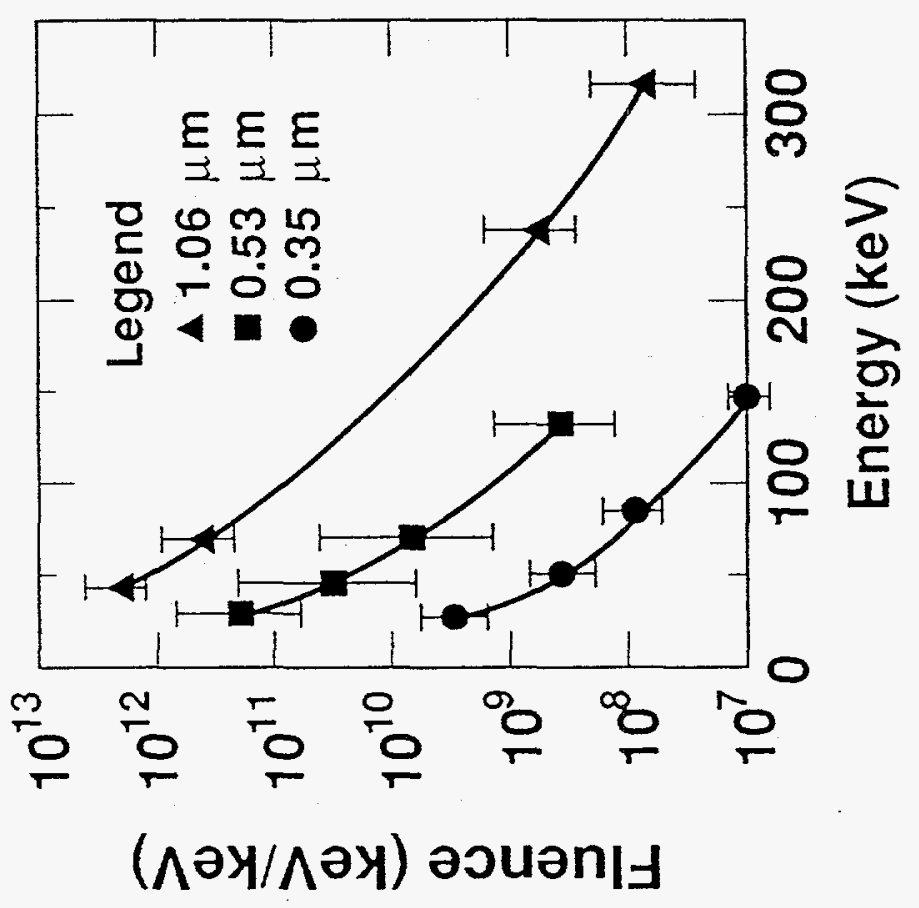

$\frac{m}{5}$
$\frac{1}{0}$
$\frac{5}{5}$
$\frac{5}{5}$
$\frac{5}{0}$ 


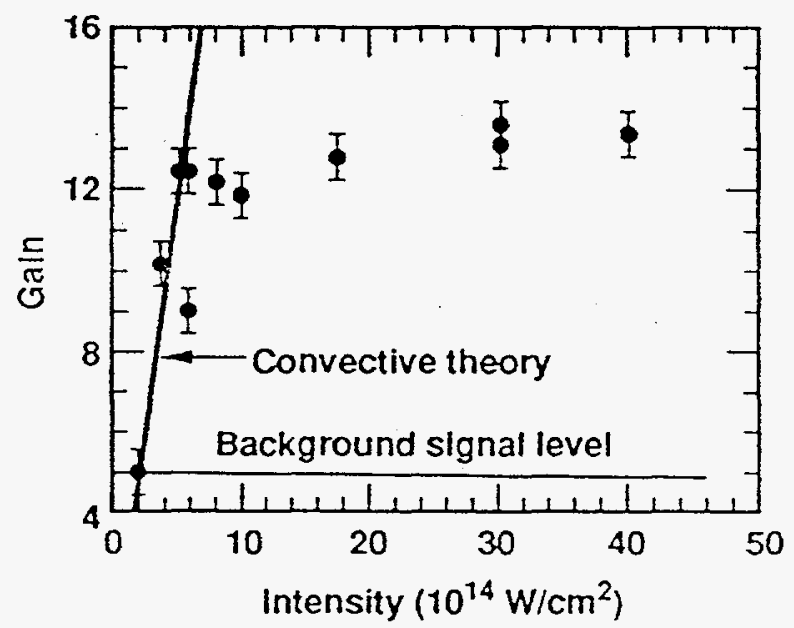

$50-00 \cdot 1292-3724$ pub

$-F$ 

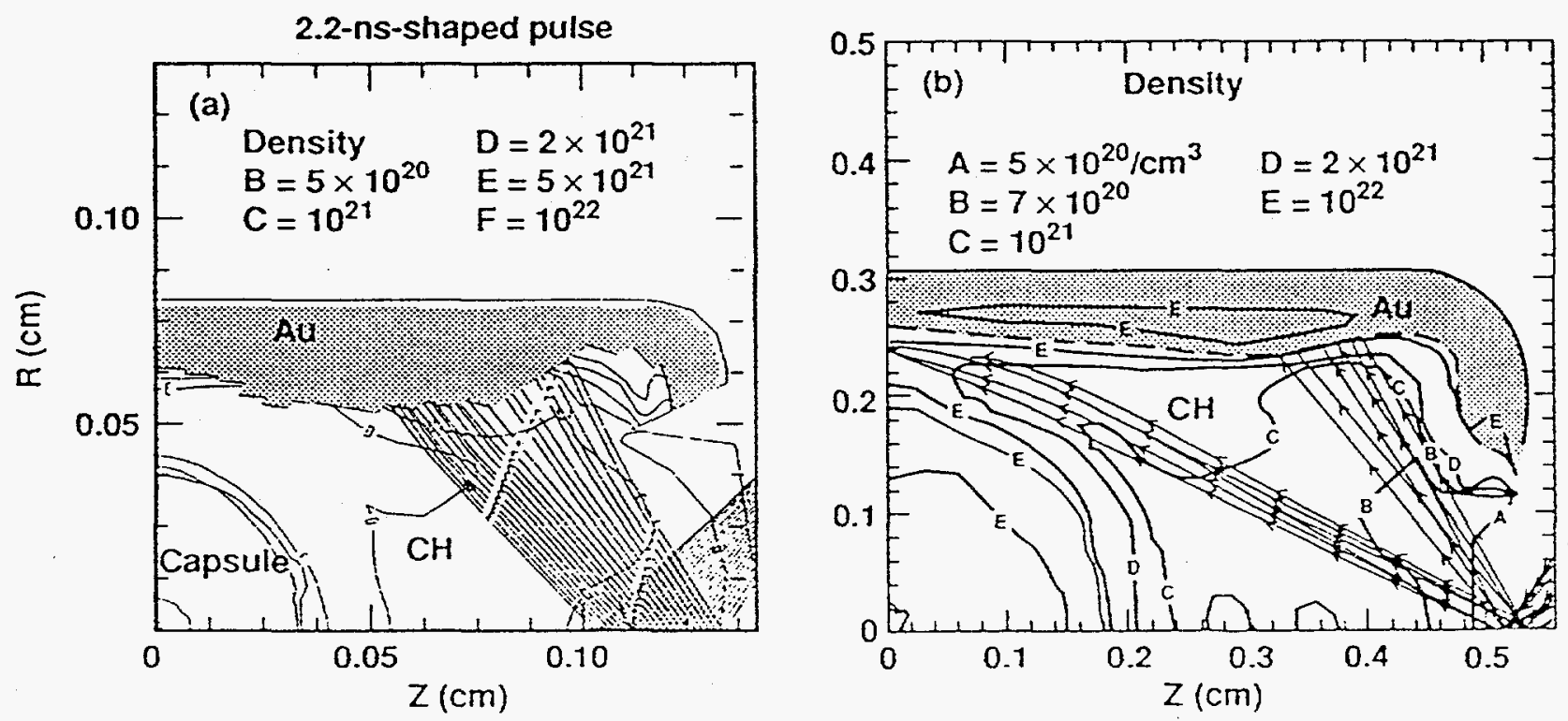

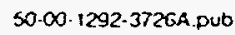




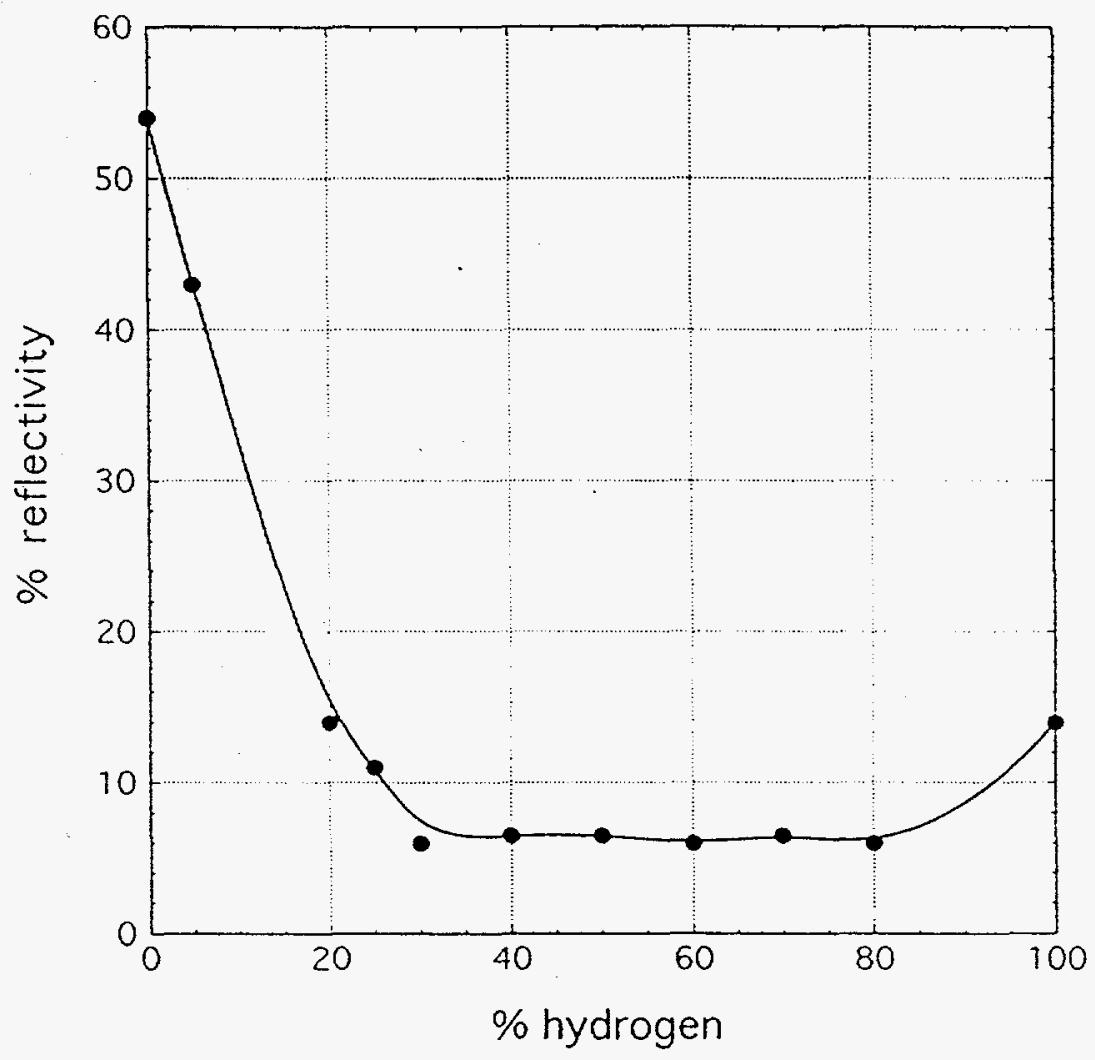

FIG 


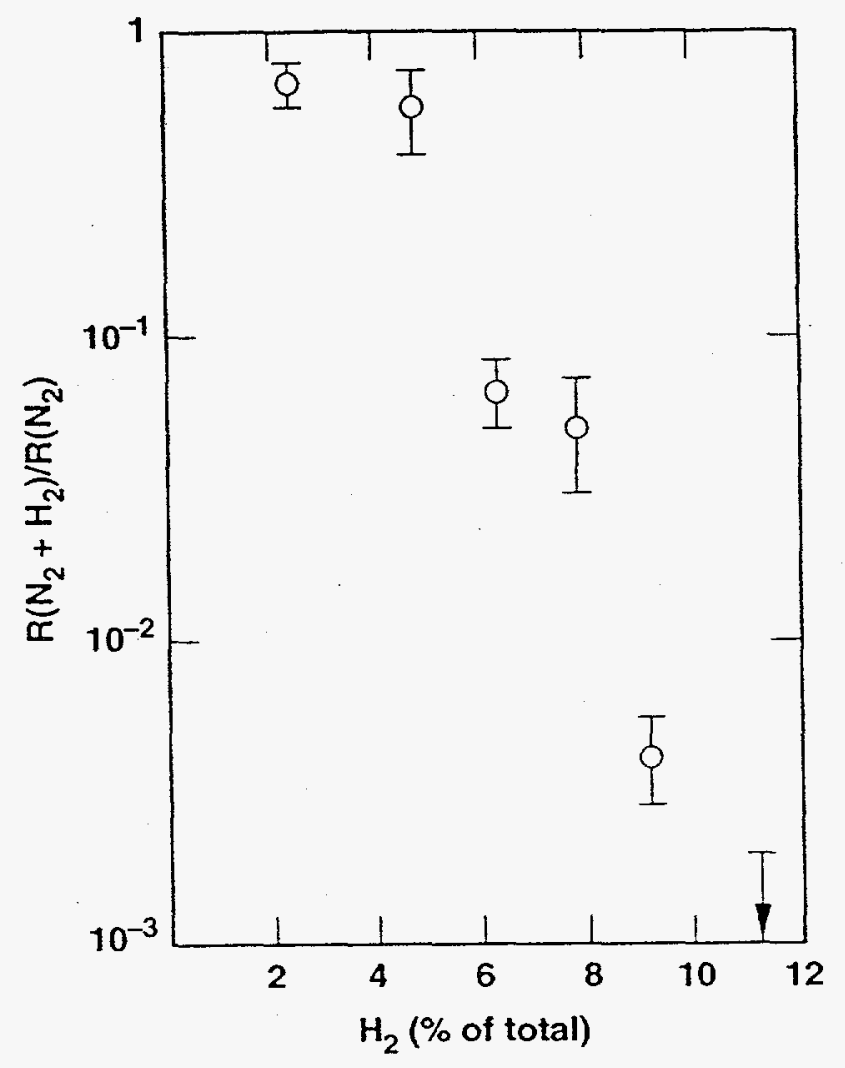

50-01-0994-3429pb01

2OWLKGgh

Fis 9 


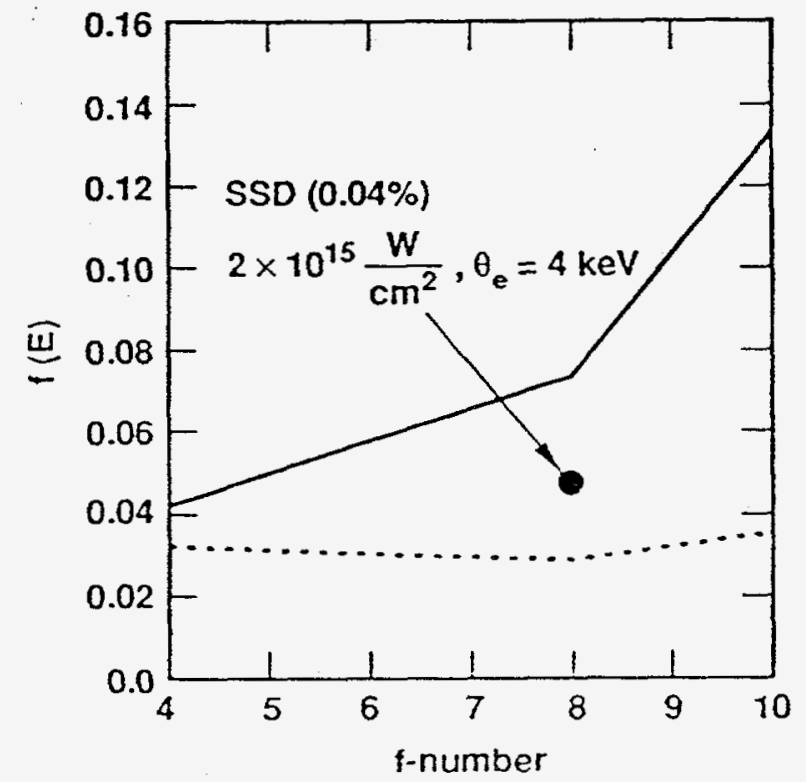

50-00-1292-3722.pub 


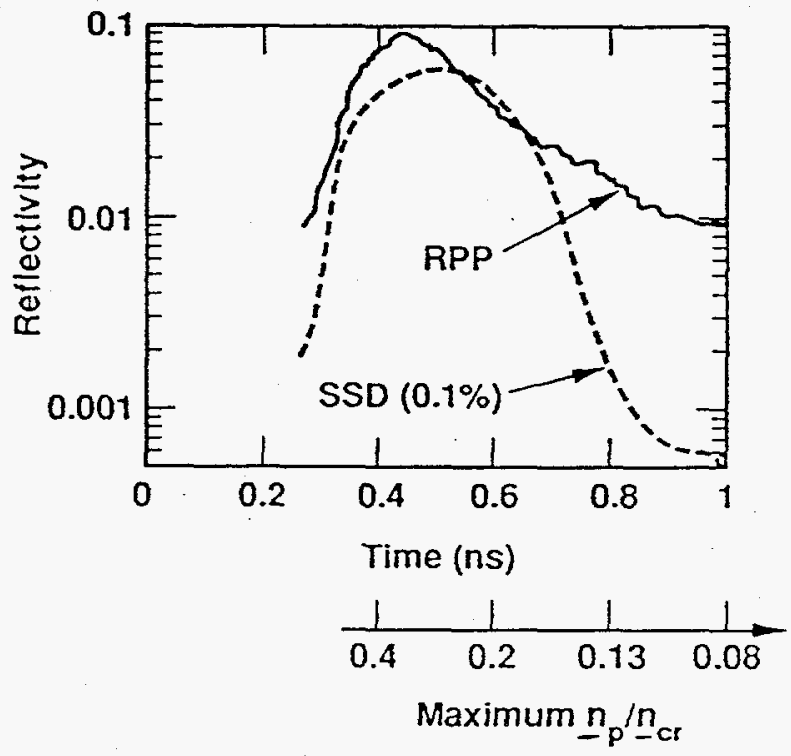

50-00-129?-3900.put 


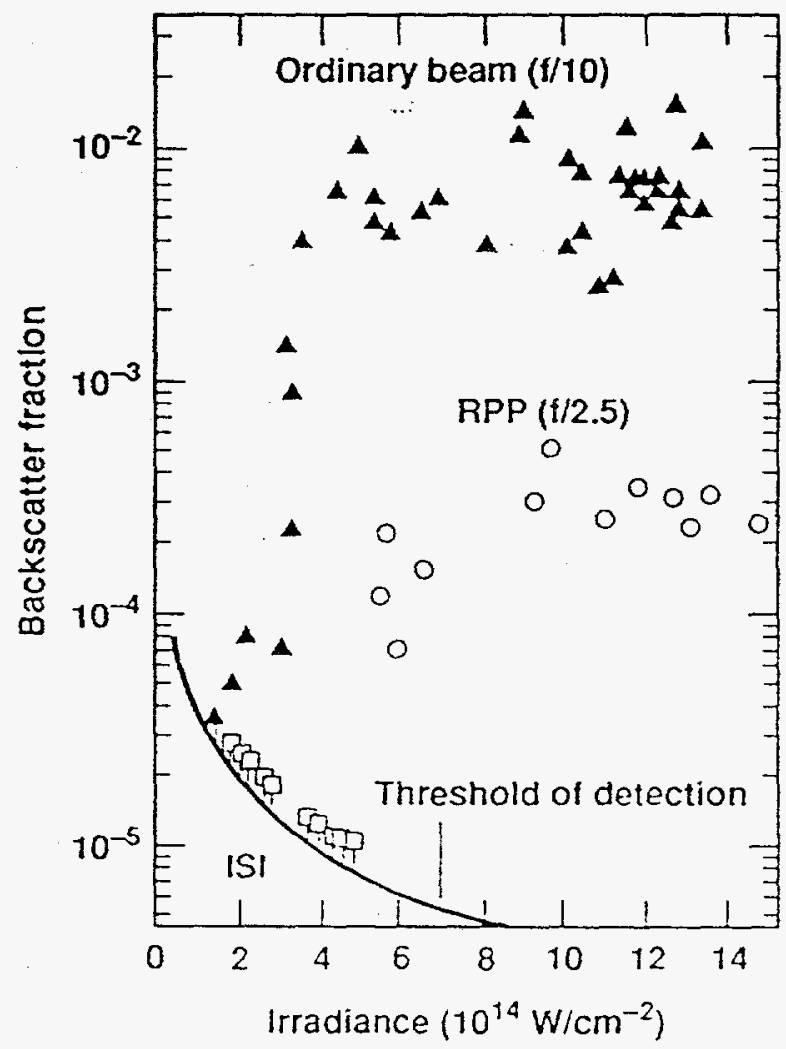

50.00-0390-1727A.PUD

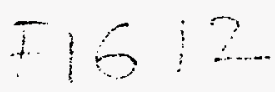




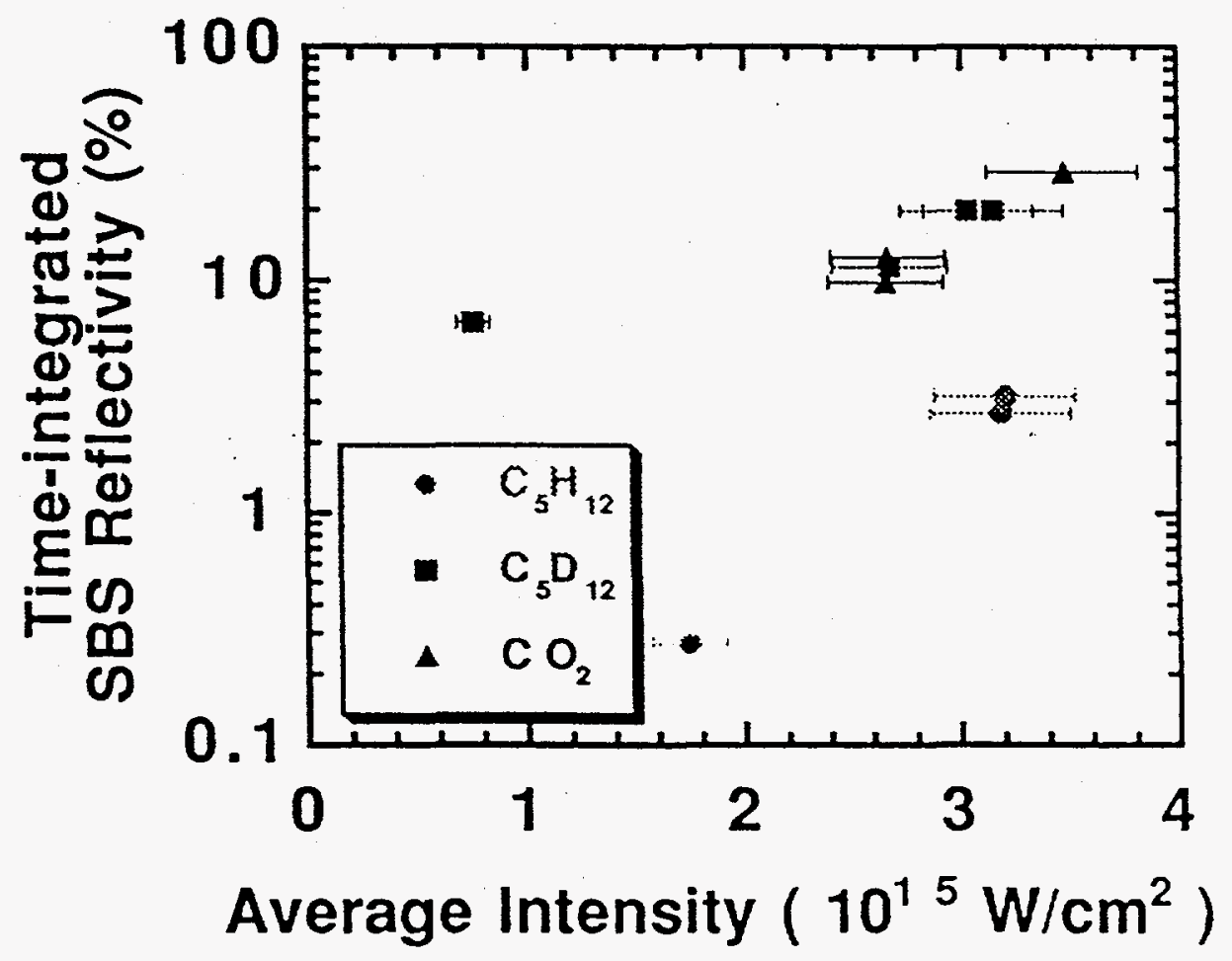

